\title{
A multi-optimization model for the design of hydrogen supply chains.
}

\author{
Miao Li ${ }^{1,2,3^{*}}$, Baolian $\mathrm{Yi}^{2}$, Pingwen Ming ${ }^{4}$, Danmin Xing ${ }^{1}$, Changfu Liu ${ }^{l}$ \\ ${ }^{1}$ Sunrise Power CO.,LTD. Dalian,116085,PR China. \\ ${ }^{2}$ Dalian institute of chemical physics, Chinese academy of sciences. Dalian, 116023,PR China \\ ${ }^{3}$ School of Mechanical Engineering and Automation, Dalian Polytechnic University, Dalian, 116034, China; \\ ${ }^{4}$ Sch Automot Studies, Tongji Univ, 4800 Caoangong Rd, Shanghai 200092, PR China
}

\begin{abstract}
This paper presents a multi-objective optimization models which operating as time processes (2025-2035) for the design of hydrogen supply chain. The feasibility of the models are illustrated through a detail case study of Dalian, China. Furthermore, the case is evaluated with the total daily costs and $\mathrm{CO}_{2}$ emissions reduction constraints. The results show that with the increase of hydrogen demand, considering the environmental factors, SMR are mostly applied in hydrogen production link. Shahekou and Pulandian grids are suitable for the constructions of hydrogen production units. Tank trucks are the main modes of hydrogen transportation. The proposed design models can provide policy-makers with the selection of infrastructures pathways for strategic dynamic hydrogen development planning.
\end{abstract}

\section{Introduction}

At present, developed countries have incorporated hydrogen energy into their energy development strategies and regarded it as one of the key sources of energy to achieve low-carbon social development goals. China has also attached great importance to the development of hydrogen energy industry. Great progress has been made in technological innovation and application of terminal products and infrastructure construction. Hydrogen fuel cell for vehicle will be an important development direction of hydrogen utilization in the future. Nowadays, developed countries such as Europe, America and Japan have supported the research, development, demonstration and commercial application of fuel cell technology through formulating medium and long-term development plans. However, the source of hydrogen is a key issue to the commercialization of fuel cell vehicles. Hydrogen production, storage and transportation and infrastructure construction are important factors for fuel cell vehicles from research demonstration to small-scale demonstration operation, to preliminary commercialization and application, and finally to large-scale application [1-5]. Therefore, the construction of hydrogen energy industry chain is very important.

This paper presents a multi-objective optimization models which operating as time processes (2025-2035) for the design of hydrogen supply chain. The models are illustrated through a detail case study of Dalian, in China. And the case is evaluated with the total daily costs and $\mathrm{CO}_{2}$ emissions reduction constraints.

\section{The optimization model of hydrogen supply chain ${ }^{[6]}$}

The hydrogen industry chain covers hydrogen production, storage, transportation and application. The cost model of hydrogen production is calculated as follows:

$$
P C=\sum_{g, p, f}\left(\frac{P C C_{p, f} N P F_{g, p, f} A F_{p}}{O P}+P R_{g, p, h, f} U P C_{p, f}\right)
$$

Where $P C C_{p f}$ is the capital cost of establishing plant type $\mathrm{p}$ producing product form $\mathrm{f}$ and $U P C_{p f}$ is the unit production cost for product. $N P F g p f$ denotes the number of production plant. $A F_{p}$ is an annuity factor for facility $\mathrm{p}$, OP represents the operating period.

The fuel $(F C)$, labor $(L C)$ and maintenance $(M C)$ costs associated with the delivery distance can affect the operation costs. Therefore, the total delivery cost is represented as:

$T C=\sum_{g, g^{\prime}, t, f}\left(\frac{T C C_{t, f} N T F_{g, g^{\prime}, t, f} A F_{t}}{O P}\right)+F C+L C+M C$

Where $T C C_{t f}$ denotes the capital cost of transport mode $t$ for the distribution of hydrogen in form $f . N T F_{g g}$ ' $f$ is the number of transport mode. $A F_{t}$ is an annuity factor for transport mode $t$.

$$
N T F_{g, g^{\prime}, t, f} \geq Q_{g, g^{\prime}, t, f}\left(\frac{2 D i s_{g, g^{\prime}, t}}{S P_{t}}+L U T_{t}\right) /\left(T M A \cdot T C a P_{t, f}\right)
$$

\footnotetext{
* Corresponding author: limiaolaile@163.com
} 
Disgg't is the average distance travelled by transportation mode t. $S P_{t}$ denotes the average speed. $L U T_{t}$ is the load/unload time. $T M A_{t}$ is availability time. TCap $_{t f}$ is the capacity .

$$
F C=\sum_{g, g^{\prime}, t, f} \frac{F P_{t}}{F E_{t}} 2 \operatorname{Dis}_{g, g^{\prime}, t} Q_{g, g^{\prime}, t, f} / \operatorname{TCap}_{t, f}
$$

$F P_{t}$ represents the fuel price, $F E T_{t}$ is the fuel economy.

$$
L C=\sum_{g, g^{\prime}, t, f} D W_{t} Q_{g, g^{\prime}, t, f}\left(\frac{2 D i s_{g, g^{\prime}, t}}{S P_{t}}+L U T_{t}\right) / \operatorname{TCap}_{t, f}
$$

Where $D W_{t}$ represents driver wage, who drives transportation mode $t$.

$$
M C=\sum_{g, g^{\prime}, t, f} M E_{t} 2 \operatorname{Dis}_{g, g^{\prime}, t} Q_{g, g^{\prime}, t, f} / \operatorname{TCap}_{t, f}
$$

$M E_{t}$ denotes the maintenance cost for transportation mode $_{t}$.

The price for the primary energy source is calculated as follow:

$$
\begin{aligned}
& P E S=\sum_{g^{\prime}, g, p, e}\left(\text { PESAv }_{g^{\prime}, g, p, e}\left(\text { ESDIS }_{e} \text { Dis }_{g^{\prime}, g}+\text { ESCOSt }_{e}\right)\right) \\
& +\sum_{g, p, e} P E S I_{g, p, e} \text { ESICost }_{e}
\end{aligned}
$$

Where ESICost $t_{e}$ represents the energy source $e$ import price, ESCost $t_{e}$ denotes local generated energy source $e$ price, $E S D I S_{e}$ is the delivery price for energy source $e$, and $D i s_{g^{\prime}, g}$ is the distance between grid point.

The storage facility can store hydrogen in different types depending on its physical form. Each storage facilities involve type, number, capacities and locations decision variable.

$$
\begin{aligned}
& S C=\sum_{g, s, f}\left(\frac{S C C_{s, f} N S F_{g, s, f} A F_{s}}{O P}+U S C_{s, f} \cdot S T_{g, f}\right) \\
& S T_{g f}=\beta D T_{g f} \forall f, g
\end{aligned}
$$

Where $S C C_{s f}$ denotes the capital cost of establishing storage type storing product form $f$, and $U S C_{s f}$ is the unit storage cost. $N S F_{g s f}$ is the number of storage facility. $A F_{s}$ is annuity factor for storage facility $s . S T_{g f}$ is the total average inventory of product form $f$ in grid g. $\beta$ is storage holding period-average number of days worth of stock.

\section{A multi-objective optimization model}

The multi-objective optimization includes economic and environmental effects in the following:

$$
C E_{\text {optimal }}=\min \left\{\lambda_{1} \text { TotalCost }+\lambda_{2} \text { TotalCO }\right\}_{2}
$$

$\lambda_{1}$ and $\lambda 2$ is the weight factor.
The first objective function consists of the production (PC), storage (SC), and transportation costs (TC) and the energy source costs (PES) are included as given by:

$$
\text { TotalCost }_{\text {optimal }}=\min \{P C+S C+T C+P E S\}
$$

The total daily $\mathrm{CO} 2$ emissions are associated with production plant, storage facility and transportation of HSC network as follows:

$$
\mathrm{CO}_{2 \text { optimal }}=\min \left\{\mathrm{PCO}_{2}+\mathrm{SCO}_{2}+\mathrm{TCO}_{2}\right\}
$$

And the total $\mathrm{CO}_{2}$ emission generated by the HSC operation $\left(\mathrm{CO}_{2} \mathrm{E}\right)$ is given as:

$$
C \mathrm{O}_{2} E=\mathrm{CO}_{2} E P_{g, p, f}+C \mathrm{O}_{2} E T_{g, g^{\prime}, t, f}+C \mathrm{O}_{2} E S_{g, s, f}
$$

Where $\mathrm{CO}_{2} E P_{g p f}$ denotes the $\mathrm{CO}_{2}$ emission generated by the production facility, $\mathrm{CO}_{2} E T_{\mathrm{g}, \mathrm{g}, \mathrm{t}, \mathrm{f}}$ is the $\mathrm{CO}_{2}$ emission generated by the $t$ th distribution mode and $\mathrm{CO}_{2} E S_{g s f}$ is the total $\mathrm{CO}_{2}$ emission produced by storage sites.

In above equation,

$$
\begin{gathered}
C O_{2} E P_{g p f}=P T_{g p f} C E F_{p f} \forall g, p, f \\
C O_{2} E T_{g, g^{\prime}, t, f}=\sum_{g, g^{\prime}, t, f} 2 D i s_{g, g^{\prime}, t, f} N T F_{g, g^{\prime}, t, f} G E T_{t} \\
C O_{2} E S_{g, s, f}=\sum_{\mathrm{g}, s, f} S T_{g, s, f} G E S_{f}
\end{gathered}
$$

$C E F_{\text {pf }}$ is the $\mathrm{CO}_{2}$ emission factor for each hydrogen technology. $T E F_{t}$ is the emission factor associated to transport mode $t$. GES is the amount of $\mathrm{CO}_{2}$ emission in the form $f$ in storage $s$.

\section{An optimization analysis-Dalian in China}

Dalian was taking as a detail cases in this study, which was divided into 9 grids, including Zhongshan, Xigang, Shahekou, Ganjingzi, lvshunkou, Jinzhou, Wafangdian, Pulandian, Zhuanghe. Hydrogen can be produced in each grid through production facilities to satisfy its hydrogen demand. The remaining hydrogen can be transported to other grids through the transportation network. In this paper, the fuel cell vehicle is assumed to be a proton exchange membrane fuel cell vehicle.The number of fuel cell vehicles was estimated based on the year-to-year permeability of FCEV. A multi-objective optimization model was established with the lowest economic cost and environmental emission constraints.

On the basis of fully considering the energy resources of Dalian and the characteristics of each hydrogen production technology, four hydrogen production units were selected in this study: steam methane reforming (SMR), coal gasification (CG), hydro-electrolysis (WE) and biomass gasification (BG). 
The optimization results in 2025 are shown in Table 1: In order to meet the hydrogen demand, 16 hydrogen production plants should be built in Dalian, and they are mainly based on SMR hydrogen production technologies. Furthermore, the production facilities optimized to be constructed in these grids are all small scales.

Table 1 Hydrogen production optimization for HSC network design 2025

\begin{tabular}{ccccc}
\hline Grid & Technology & Scale & Number & $\begin{array}{c}\text { Amount( } \\
\text { kg d-1) }\end{array}$ \\
\hline Zhongshan & SMR & Small & 1 & 7991 \\
Xigang & SMR & Small & 1 & 6403 \\
Shahekou & SMR & Small & 2 & 14237 \\
Ganjinzi & SMR & Small & 2 & 19407 \\
Lvshunkou & SMR & Small & 1 & 4542 \\
Jinzhou & SMR & Small & 2 & 14205 \\
Wafangdian & SMR & Small & 3 & 20411 \\
Pulandian & SMR & Small & 2 & 18669 \\
Zhuanghe & SMR & Small & 2 & 18406 \\
\hline
\end{tabular}

It can be also seen from the Table 1 that each grid has its own hydrogen production plant, which can completely satisfy their hydrogen demand. Consequently, there is no hydrogen transported between grids. Furthermore large-amount production facilities are optimized to be constructed in Wafangdian grid.

The optimization results of 2030 in Table 2 and Table 3 show that hydrogen production plants need to be constructed in Shahekou and Pulandian grid of Dalian, . With the increase of hydrogen demand, the production plants are mostly medium scale. The result is obviously different from the plans of 2025, which is mainly small scale plants. Furthermore, considering the environmental factors, the selection of SMR hydrogen production technology is the main hydrogen production technology in all the grids. The reason is that $\mathrm{CG}$ has high $\mathrm{CO}_{2}$ emission factor, although it can be produced easily from economic viewpoints. Additionally, hydrogen transport facility is mainly tank truck. The amount of hydrogen transportation are obviously increased and the number of transport facilities are 8 in all grids. This means that with the increase of hydrogen demand, the comprehensive evaluation of economic and environmental can be obtained through hydrogen allocation between each grids.

Table 2 Hydrogen production optimization for HSC network design 2030

\begin{tabular}{ccccc}
\hline Grid & Technology & Scale & Number & $\begin{array}{c}\text { Amount } \\
\text { kg d-1) }\end{array}$ \\
\hline Shahekou & SMR & Medium & 1 & 142853 \\
Pulandian & SMR & Medium & 1 & 93989 \\
\hline
\end{tabular}

Table 3 Hydrogen Transportation optimization for HSC network design 2030

\begin{tabular}{ccccc}
\hline From & To & Type & Number & $\begin{array}{c}\text { Amoun } \\
\mathrm{t}(\mathrm{kg} \mathrm{d}- \\
1)\end{array}$ \\
\hline Shahekou & Zhongshan & Tank truck & 1 & 16155 \\
& Xigang & Tank truck & 1 & 12944 \\
& Ganjingzi & Tank truck & 1 & 39235 \\
& Lvshunkou & Tank truck & 1 & 8326 \\
& Wafangdian & Tank truck & 2 & 37410 \\
Pulandian & jinzhou & Tank truck & 1 & 26037 \\
& zhuanghe & Tank truck & 1 & 33736 \\
\hline
\end{tabular}

The optimization results of 2035 are shown as Table 4 and Table 5: in order to meet the hydrogen demands, four hydrogen production units should be constructed in Dalian. And Shahekou, Ganjingzi, Jinzhou and Pulandian grids are suitable for the construction of medium-scale hydrogen production units. The hydrogen production technologies are mainly SMR technologies.

Table 4 Hydrogen production optimization for HSC network design 2035

\begin{tabular}{ccccc}
\hline Grid & Technology & Scale & Number & $\begin{array}{c}\text { Amount( } \\
\text { kg d-1) }\end{array}$ \\
\hline Shahekou & SMR & Medium & 1 & 104874 \\
Ganjingzi & SMR & Medium & 1 & 71305 \\
Jinzhou & SMR & Medium & 1 & 101931 \\
Pulandian & SMR & Medium & 1 & 117621 \\
\hline
\end{tabular}

It can be also seen that the hydrogen transportation and allocation between the grids can not satisfy the increasing hydrogen demand. Therefore the number of hydrogen transportation units are increased compared with that in 2030. Hydrogen transport facility is mainly tank truck which is consistent with 2030. The hydrogen can be transported from Shahekou grid, Ganjinzi grid, Jinzhou grid and Pulandian grid to other areas. In particular, the amount of hydrogen which transported from Jinzhou grid to Wafangdian grid is the largest.

Table 5. Hydrogen Transportation optimization for HSC network design 2035

\begin{tabular}{ccccc}
\hline From & To & Type & Number & $\begin{array}{c}\text { Amount } \\
(\mathrm{kg} \mathrm{d}-1)\end{array}$ \\
\hline Shahekou & Zhongshan & Tank truck & 1 & 26994 \\
& Xigang & Tank truck & 1 & 21626 \\
& Ganjingzi & Tank truck & 1 & 4081 \\
& Lvshunkou & Tank truck & 1 & 4081 \\
Ganjingzi & Lvshunkou & Tank truck & 1 & 9830 \\
Jinzhou & Wafangdian & Tank truck & 1 & 58427 \\
Pulandian & Wafangdian & Tank truck & 1 & 4081 \\
& Zhuanghe & Tank truck & 2 & 56368 \\
\hline
\end{tabular}




\section{Conclusion}

In this paper, a multi-optimization model operating as time processes (2025-2035) for hydrogen infrastructure plan has been proceeded in Dalian, China. The two objectives considered were cost and environmental impact. Four types of technologies to produce hydrogen were evaluated, namely steam methane reforming (SMR), coke oven gas hydrogen production (COG), hydro-electrolysis (WE) and biomass gasification (BG). From the economic and environmental view point, this study showed that, SMR are mostly selected and applied in hydrogen production plant. While Shahekou and Pulandian grids are suitable for the construction of hydrogen production units. Meanwhile, with the increase of hydrogen demand, the multi-objective optimization can be obtained through hydrogen allocation between each grids. Additionally, tank trucks are the main modes of hydrogen transportation.

\section{Acknowledgement}

The authors gratefully acknowledge the financial support from the National Natural Science Foundation of China (71603035) and Foundation of Liaoning province (J2019047).

\section{References}

1. Jenn-Jiang Hwang. Sustainability study of hydrogen pathways for fuel cell vehicle applications. Renewable and Sustainable Energy Reviews 19 (2013) 220-229.

2. Mengyu Li, Xiongwen Zhang, Guojun Li. A comparative assessment of battery and fuel cell electric vehicles using a well-to-wheel analysis. Energy 94 (2016) 693-704.

3. Noriko Behling, Mark C. Williams, Shunsuke Managia. Fuel cells and the hydrogen revolution: Analysis of a strategic plan in Japan. Economic Analysis and Policy 48 (2015) 204- 221.

4. Dawei Wang, Nada Zamel, Kui Jiao, Yibo Zhou, Shuhai Yu, Qing Du, Yan Yin. Life cycle analysis of internal combustion engine, electric and fuel cell vehicles for China. Energy 59 (2013) 402-412.

5. Dominković D, Bačeković I, Pedersen AS, Krajačić G. The future of transportation in sustainable energy systems: opportunities and barriers in a clean energy transition. Renew Sustain Energy Rev 2017.

6. Anton Ochoa Bique, Leonardo K.K. Maia, Fabio La Mantia, Dabide Manca, Edwin Zondervan. Balancing costs, safety and $\mathrm{CO} 2$ emissions in the design of hydrogen supply chains.Computers and Chemical Engineering 129 (2019) 106493. 